\title{
Pustular Rash in Crohn's Patient on Ustekinumab Raises Concern for Drug-Induced Paradoxical Psoriasis
}

\author{
Mitra Barahimi Scott Lee Kindra Clark-Snustad \\ Department of Gastroenterology, University of Washington, Seattle, WA, USA
}

\author{
Keywords \\ Crohn's disease $\cdot$ Rash $\cdot$ Treatment
}

\begin{abstract}
We report the case of a 51-year-old male with Crohn's disease (CD) who developed a reproducible pustular rash after ustekinumab (UST) administration. The patient first presented with a pustular rash on his hands, body, extremities, and scalp starting 5 weeks after his initial weight-based UST induction. The rash resolved spontaneously, then recurred 4 weeks after his first subcutaneous maintenance dose of UST $90 \mathrm{mg}$. Biopsy of the affected area demonstrated subcorneal pustular dermatosis (SPD). UST was discontinued and the rash resolved. Unfortunately, the patient experienced clinical recurrence of $C D$, and given prior failure of multiple CD medications, UST was restarted with premedication. Two weeks after UST reinduction, the rash recurred, though less severe. Given improvement in CD symptoms, UST was continued and the rash managed with topical corticosteroids. This is the first case of druginduced SPD associated with UST. One case report has previously described de novo pustular psoriasis associated with UST in a patient with CD and enteropathic arthritis. Notably, SPD and pustular psoriasis can be histologically indistinguishable. The development of a paradoxical psoriasiform rash is thought to be one of the few dose and duration dependent side effects of TNF-antagonist therapy but has not previously been established as a side effect of UST. This case demonstrates a new potential side effect of UST.
\end{abstract}

\section{Introduction}

Crohn's disease (CD) is a chronic autoimmune disorder treated with long-term immunosuppression. Ustekinumab (UST) is an interleukin 12/23 inhibitor that is FDA approved for CD and ulcerative colitis (UC), as well as for psoriasis and psoriatic arthritis. TNF-antagonist 
Fig. 1. Pustular rash involving patient's face which developed 5 weeks after IV weight-based induction with UST $520 \mathrm{mg}$ for treatment of CD. CD, Crohn's disease; UST, ustekinumab.

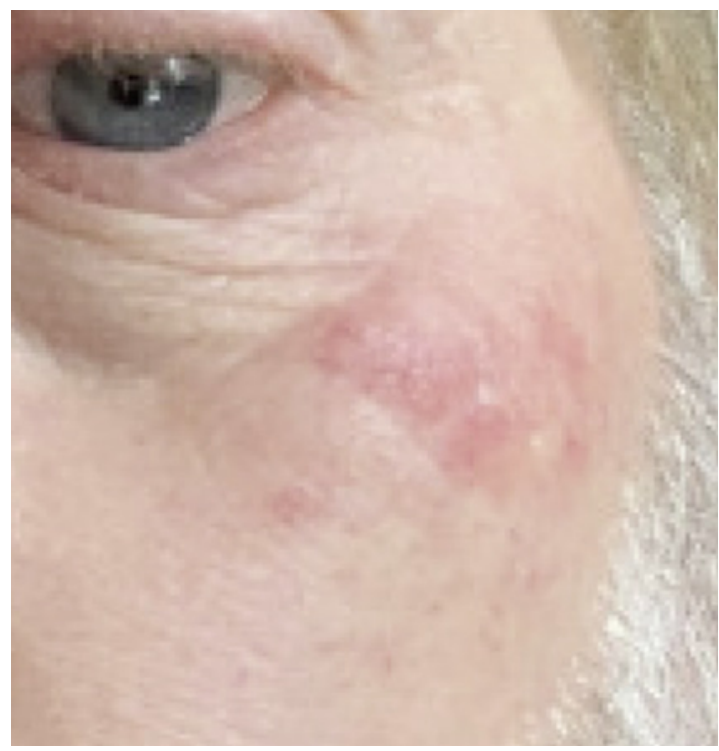

therapy is another treatment for $\mathrm{CD}$ and has been associated with the development of a dose and duration-dependent paradoxical psoriasiform rash. Previously, UST had not been associated with this adverse effect. We present the case of de novo pustular psoriasis in a patient with CD treated with UST. The patient in our case presented with a pustular rash after UST IV induction. The rash recurred after a second UST dose, resolved upon UST discontinuation, then recurred upon UST re-induction. Our case suggests a possible new adverse event associated with UST therapy.

\section{Case Report/Case Presentation}

We report the case of a 51-year-old male with long-standing CD who developed biopsyproven relapsing/remitting subcorneal pustular dermatosis (SPD) after UST treatment. The patient was initially diagnosed with UC in the early 1990s and underwent ileoanal pouch anastomosis in 2014. Postoperatively he developed Crohn's ileitis prompting initiation of biologic therapy in 2015. He failed TNF-antagonist therapy with adalimumab due to worsening enteropathic arthritis and developed secondary loss of response to infliximab and methotrexate. Given both luminal disease activity and enteropathic arthritis, he was switched to UST in 2017 and remained on varying doses of subcutaneous methotrexate managed by his rheumatologist. Following induction with IV, weight-based UST $520 \mathrm{mg}$ in November 2017, he experienced significant clinical improvement in CD symptoms including improved energy, joint pain, bowel frequency, and stool consistency. Approximately, 5 weeks after the UST infusion, he developed a pustular rash on his hands, body, face, extremities, and scalp as shown in Figure 1. The rash improved one week later, then resolved spontaneously. He subsequently administered his first maintenance injection with UST $90 \mathrm{mg}$ subcutaneous 8 weeks after his induction dose in January 2018. His CD symptoms again improved, and then approximately 4 weeks later, he had recrudescence of the pustular rash, primarily involving his palms (shown in Fig. 2). Subsequent biopsy of the left and right palm demonstrated SPD. As the adverse event was temporally associated with UST, we recommended UST discontinuation (last dose January 2018). The rash resolved spontaneously and did not recur while off UST. Unfortunately, the patient experienced clinical recurrence of CD and arthritis, and given 
Fig. 2. Recurrence of patient's pustular rash involving the palms 4 weeks after first maintenance injection with UST $90 \mathrm{mg}$ SC for treatment of CD. CD, Crohn's disease; SC, subcutaneous.

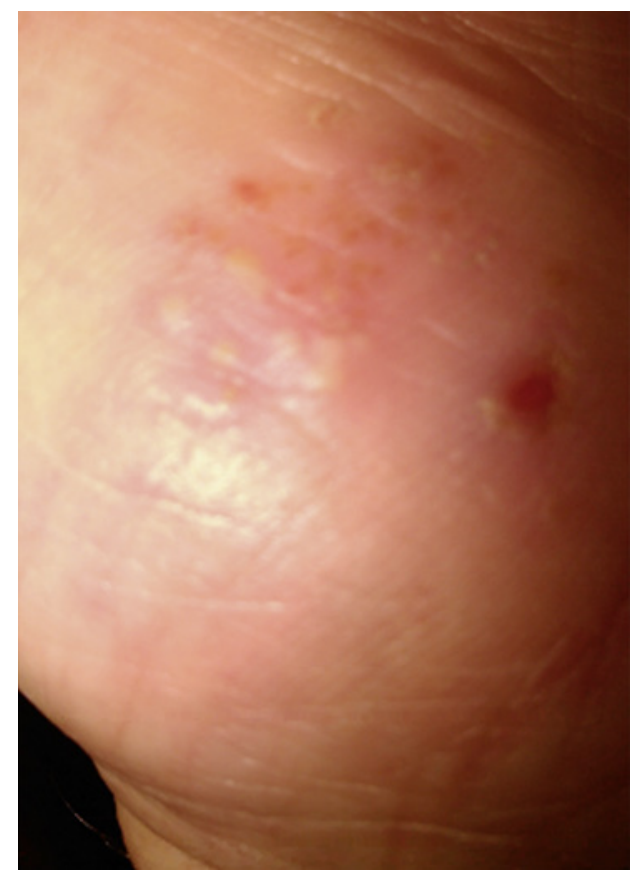

failure of multiple CD therapies, UST was restarted with IV, weight-based infusion of UST 520 mg in April 2020, administered with premedication (acetaminophen $650 \mathrm{mg}$ and diphenhydramine $25 \mathrm{mg}$ prior to infusion and prednisone $40 \mathrm{mg}$ day prior, day of, and day after UST). Two weeks after re-induction, the patient had recurrence of the pustular rash on his back, though with less severe presentation. Given improvement in the patient's refractory CD, UST was continued and the rash managed with topical corticosteroids.

\section{Discussion/Conclusion}

This is the first case of drug-induced SPD associated with UST. SPD is a rare skin disorder characterized by sterile pustules in the most superficial layer of the skin. The etiology of SPD is unclear but it has been associated with cutaneous and other systemic autoimmune conditions including pyoderma gangrenosum, Sweet syndrome, CD [1], and rheumatoid arthritis. Histologically, SPD is similar to and can be indistinguishable from pustular psoriasis [2]. UST is thought to mechanistically work via inhibition of interleukin $12 / 23$ and in addition to being FDA approved for CD and UC is also approved for the treatment of psoriasis and psoriatic arthritis. One case report has described de novo pustular psoriasis associated with UST in a patient with CD and enteropathic arthritis [3]. Interestingly, several cases describing paradoxical flare of pustular psoriasis in patients with underlying psoriasis have also been reported [4-8]. The development of a paradoxical psoriasiform rash is thought to be one of the few dose and duration dependent side effects of TNF-antagonist therapy but has not been established as a side effect of UST. The paradoxical psoriasiform rash seen with anti-TNF therapy tends to present late in the course of treatment in contrast to this case, where our patient presented with this reproducible adverse event after his first two doses of UST. In our experience, the paradoxical psoriasiform rash associated with anti-TNF therapy can be managed about $50 \%$ of the time with the addition of methotrexate or azathioprine with topical corticosteroids [9]. Given the experience with anti-TNF related psoriasiform rash

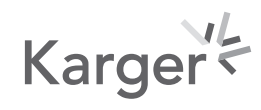


coupled with the patient's robust clinical improvement on UST, we attempted to overcome this adverse event with corticosteroid, acetaminophen, and antihistamine premedication as well as topical corticosteroid therapy. To date, with these therapies, the patient's rash is stable and manageable. Importantly, our patient had no history of psoriasis. Causality was assessed using World Health Organization criteria and determined to be probable [10]. While the patient's rash could represent native autoimmune disease such as pustular psoriasis unrelated to UST, its temporal relationship with drug administration including resolution between doses and with cessation of the drug, as well as subsequent recurrence with reintroduction of the drug, suggests it is more likely a drug-induced phenomenon.

\section{Statement of Ethics}

Written informed consent was obtained from the patient for publication of this case report and the accompanying images.

\section{Conflict of Interest Statement}

Mitra Barahimi has no conflicts of interest to declare. Kindra Clark-Snustad is a consultant for Pfizer Pharmaceuticals. Scott Lee receives grant/research support from AbbVie, UCB Pharma, Janssen, Salix Pharmaceuticals, Takeda, Celgene, Arena Pharmaceuticals, and AbGenomics and is a consultant for UCB Pharma, Cornerstones Health, Janssen, Eli Lilly and Company, Celgene, KCRN Research, Boehringer Ingelheim, Bristol-Myers Squibb, Applied Molecular Transport, Arena Pharmaceuticals, Celltrion Healthcare, Samsung Bioepis Company, and Bridge Biotherapeutics.

\section{Funding Sources}

This manuscript did not receive any funding.

\section{Author Contributions}

Mitra Barahimi, Scott Lee, and Kindra Clark-Snustad contributed equally to this article. All the authors approved final version of the article.

\section{References}

1 Delaporte E, Colombel JF, Nguyen-Mailfer C, Piette F, Cortot A, Bergoend H. Subcorneal pustular dermatosis in a patient with Crohn's disease. Acta Derm Venereol. 1992 Aug;72(4):301-2.

2 Sanchez NP, Perry HO, Muller SA, Winkelmann RK. Subcorneal pustular dermatosis and pustular psoriasis. A clinicopathologic correlation. Arch Dermatol. 1983;119(9):715.

3 Benzaquen M, Flachaire B, Rouby F, Berbis P, Guis S. Paradoxical pustular psoriasis induced by ustekinumab in a patient with Crohn's disease-associated spondyloarthropathy. Rheumatol Int. 2018 Jul;38(7):1297-9.

4 Caca-Biljanovska N, V'lckova-Laskoska M, Laskoski D. Successful management of ustekinumab-induced pustular psoriasis without therapy discontinuation. Acta Dermatovenerol Croat. 2013;21(3):202-4.

5 Hay RA, Pan JY. Paradoxical flare of pustular psoriasis triggered by ustekinumab, which responded to adalimumab therapy. Clin Exp Dermatol. 2014 Aug;39(6):751-2.

6 Gregoriou S, Kazakos C, Christofidou E, Kontochristopoulos G, Vakis G, Rigopoulos D. Pustular psoriasis development after initial ustekinumab administration in chronic plaque psoriasis. Eur J Dermatol. 2011 Jan;21(1):104-5. 
7 Suh HY, Ahn JY, Park MY, Youn JI. Exacerbation of infliximab-induced paradoxical psoriasis after ustekinumab therapy. J Dermatol. 2018 Mar;45(3):332-3.

8 Wenk KS, Claros JM, Ehrlich A. Flare of pustular psoriasis after initiating ustekinumab therapy. J Dermatolog Treat. 2012 Jun;23(3):212-4.

9 Afzali A, Wheat CL, Hu JK, Olerud JE, Lee SD. The association of psoriasiform rash with anti-tumor necrosis factor (anti-TNF) therapy in inflammatory bowel disease: a single academic center case series. J Crohns Colitis. 2014 Jun;8(6):480-8.

10 Uppsala Monitoring Centre [Internet]. The use of the WHO-UMC system for standardised case causality assessment. [cited 2020 Aug 7]. Available from: www.who.int/medicines/areas/quality_safety/safety_ efficacy/WHOcausality_assessment.pdf?ua=1. 